\title{
OPTIMAL PERIODIC OUTPUT FEEDBACK CONTROL: A CONTINUOUS TIME APPROACH
}

\author{
Luca Viganò* Marco Lovera* Andras Varga ** \\ * Dipartimento di Elettronica e Informazione \\ Politecnico di Milano \\ P.za Leonardo da Vinci 32, 20133 Milano, Italy \\ Phone: + 39 02 23993592; Fax: +39 02 23993412 \\ Email: \{vigano,lovera\}@elet.polimi.it \\ ** German Aerospace Center, DLR-Oberpfaffenhofen \\ Institute of Robotics and Mechatronics \\ D-82234 Wessling, Germany \\ Email: andras.varga@dlr.de
}

\begin{abstract}
This paper addresses the problem of optimal static output feedback control of linear periodic systems. The proposed approach, which allows to deal with both stable and unstable open loop systems, relies on a continuous-time formulation of the control problem. A nonlinear programming based approach is used to solve the underlying optimization problem. The evaluation of cost function and its derivative largely benefits of the recent advances in algorithms to solve continuous-time periodic Lyapunov equations.
\end{abstract}

Keywords: Time-varying systems, Output feedback, Optimal control

\section{INTRODUCTION}

Static output feedback (SOF) stabilization of linear time-periodic (LTP) systems is a challenging and important problem, since it represents a realistic solution for a wide class of practical applications when full state measurement is not available and the complexity of a high-order compensator needs to be avoided. In particular, even in the case of full state measurement the determination of a constant stabilising feedback gain is an important and non trivial problem. The existing literature on this problem can be briefly summarised as follows.

Stabilizability by means of SOF: the problem of determining whether a stabilizing constant or periodic memoryless controller exists is without doubt an open question. An existence condition for the periodic feedback case has been provided in (Colaneri et al., 1998).

Pole placement with SOF: (Ayels and Willems, 1993) suggested a simple algorithm for the assignment of the characteristic exponents of a controllable LTP systems, valid only for second order, discrete-time case. Other works, such as (Juan and Kabamba, 1989; Chen and Chen, 1999) introduced the concept of generalized sample and hold functions (GSHF), which makes it possible to assign multiple poles using output measurements only once for each period $T$. Nevertheless, the GSHF is unsuitable in all applications where the period is quite long, such as satellite attitude and orbit control systems.

Linear Quadratic Control with SOF: in (Calise et al., 1992), a Floquet-transformed LQ cost- 
function is considered, penalizing the response and control envelopes rather than the actual time histories. In (Varga and Pieters, 1998) the LQ performance index for discrete-time LTP systems is minimized by computing the analytical gradient and exploiting gradient descent optimization algorithms for which reliable numerical implementations have been developed (Varga, 2005a). A similar approach has been proposed in (Aliev et al., 2005), where the cost function for the discrete-time case is minimised using a gradientfree method. More recently, an LMI approach to the problem has been proposed in (Farges et al., 2006).

The aim of this paper is to derive a procedure for the practical determination of a stabilizing output feedback gain which minimizes a quadratic performance index. The results presented in this paper apply equivalently to periodic output feedback and periodic state feedback.

\section{PROBLEM STATEMENT}

Consider the linear time-periodic system

$$
\begin{aligned}
& \dot{x}=A(t) x+B(t) u \\
& y=C(t) x
\end{aligned}
$$

where $A(t) \in \mathbf{R}^{n \times n}, B(t) \in \mathbf{R}^{n \times m}, C(t) \in$ $\mathbf{R}^{p \times n}$ are periodic matrices of period $T$, and the quadratic performance index

$$
J=E\left\{\int_{t_{0}}^{\infty}\left[x^{T} Q(t) x+u^{T} R(t) u\right] d t\right\}
$$

with $Q(t) \geq 0, R(t)>0$ symmetric T-periodic matrices. Suppose that the initial condition $x_{0}$ is a random variable with zero mean and known covariance $X_{0}=\left\{x_{0} x_{0}^{T}\right\}$. The optimal output feedback control problem consists in finding the feedback matrix $F(t)$ of optimal control action

$$
u^{*}=F(t) y
$$

which minimizes the performance index $J$ of (2). The expectation $E(\cdot)$ used in (2) allows to remove any dependence of the cost function on the initial condition, hence the resulting optimal gain may be interpreted as the optimal feedback matrix in an average sense, i.e., optimal over some set of initial states. From this point of view, the covariance matrix $X_{0}$ is a design parameter which may be used by the designer who has some $a$ priori knowledge of which states of the system are likely to be perturbed (see (Levine et al., 1971)). If this information is not available, a common choice consists in assuming an initial state uniformly distributed over a unit hypersphere (i.e., $X_{0}=I$ ). On the other hand, a fully deterministic approach requires to know exactly the initial state, to set $X_{0}=x_{0} x_{0}^{T}$ and to optimize $J$ according to it
(Brobrovsky and Graupe, 1971). Under (3), the closed loop dynamic equation can be written as

$$
\begin{aligned}
\dot{x} & =A(t) x+B(t) F(t) y= \\
& =[A(t)+B(t) F(t) C(t)] x=\bar{A}(t) x,
\end{aligned}
$$

where $\bar{A}(t)=A(t)+B(t) F(t) C(t)$ represents the closed loop dynamic matrix, which is obviously periodic. Let $\Phi_{\bar{A}}\left(t, t_{0}\right)$ the transition matrix associated with $\bar{A}(t)$, satisfying the well known equation

$$
\dot{\Phi}_{\bar{A}}\left(t, t_{0}\right)=\bar{A}(t) \Phi_{\bar{A}}\left(t, t_{0}\right), \quad \Phi_{\bar{A}}\left(t_{0}, t_{0}\right)=I .
$$

Assume now that a periodic stabilizing gain $F(t)$ is known, i.e., such that $\left|\lambda_{i}(\Psi)\right|<1 \forall i$, where the monodromy matrix $\Psi=\Phi_{\bar{A}}\left(t_{0}+T, t_{0}\right)$; then, the performance index $J$ can be written as

$$
J=E\left\{\int_{t_{0}}^{\infty}\left[x^{T} \bar{Q}(t) x\right] d t\right\}
$$

where $\bar{Q}(t)=Q(t)+C(t)^{T} F(t)^{T} R(t) F(t) C(t)$. Note that since $x(t)=\Phi_{\bar{A}}\left(t, t_{0}\right) x_{0}$, (6) is equivalent to

$$
J=E\left\{\int_{t_{0}}^{\infty} x_{0}^{T} \Phi_{\bar{A}}^{T}\left(t, t_{0}\right) \bar{Q}(t) \Phi_{\bar{A}}\left(t, t_{0}\right) x_{0} d t\right\},
$$

which, defining $P(t)=\int_{t}^{\infty} \Phi_{\bar{A}}^{T}(\tau, t) \bar{Q}(\tau) \Phi_{\bar{A}}(\tau, t) d \tau$, can be expressed as

$$
\begin{aligned}
J & =E\left\{x_{0}^{T} \int_{t_{0}}^{\infty} \Phi_{\bar{A}}^{T}\left(t, t_{0}\right) \bar{Q}(t) \Phi_{\bar{A}}\left(t, t_{0}\right) d t x_{0}\right\}= \\
& =E\left\{x_{0}^{T} P_{0} x_{0}\right\}=\operatorname{tr}\left(P_{0} X_{0}\right),
\end{aligned}
$$

where the periodic matrix $\mathrm{P}(\mathrm{t})$ satisfies a standard Periodic Lyapunov Differential Equation (PLDE), in the so-called adjoint form, i.e.,

$$
-\dot{P}(t)=\bar{A}(t)^{T} P(t)+P(t) \bar{A}(t)+\bar{Q}(t) .
$$

\section{PERFORMANCE INDEX MINIMIZATION}

The minimization of (7) can be carried out using steepest descent methods, provided that an analytical expression for the gradient of (7) with respect to the coefficients of $F$ is available. The problem of determining an initial stabilizing matrix $F_{0}$ must also be considered, if one aims at developing a design technique which can work also for open-loop unstable systems. In the following, necessary conditions for optimality (and therefore the required gradient expression) will be developed. In particular, since the case of constant gain is more interesting for applications, the hypothesis $\dot{F}=0$ will be assumed, but it is clear that structured, time-periodic gains (represented by, e.g., Fourier series expansions) can be optimised using the same approach by means of a simple reformulation of the control problem, as illustrated in the example in Section 5. In the following, the explicit time dependence of all variables will be 
omitted for the sake of readability. Recalling (6), the expression to be minimized is given by

$$
J=E\left\{\int_{t_{0}}^{\infty}\left[x^{T} \bar{Q}(t) x\right] d t\right\} .
$$

Following the approach described in (Knapp and Basuthakur, 1972), let us introduce the Hamiltonian function

$$
\begin{aligned}
H & =x^{T} \bar{Q} x+\lambda_{x}^{T} \dot{x}+\Lambda_{F}^{T} \dot{F}=x^{T} \bar{Q} x+\lambda_{x}^{T} \bar{A} x= \\
& =\operatorname{tr}\left[\bar{Q} x x^{T}+\bar{A} x \lambda_{x}^{T}\right]
\end{aligned}
$$

with

$$
\begin{aligned}
\dot{\lambda}_{x}= & -\frac{\partial H}{\partial x}=-2\left[Q+C^{T} F^{T} R F C\right] x+ \\
& -[A+B F C]^{T} \lambda_{x}, \\
\dot{\Lambda}_{F}= & -\frac{\partial H}{\partial F}=-\frac{\partial}{\partial F} \operatorname{tr}\left[\left(Q+C^{T} F^{T} R F C\right) x x^{T}+\right. \\
& \left.+(A+B F C) x \lambda_{x}^{T}\right] .
\end{aligned}
$$

where $\Lambda_{x}(\infty)=0, \Lambda_{F}(\infty)=0$. Making use of the derivation rules for the trace operator employed in (Varga and Pieters, 1998), the equation for the dynamics of the $\Lambda_{F}$ matrix turns into

$$
\dot{\Lambda}_{F}=-2 R F C x x^{T} C^{T}-B^{T} \lambda_{x} x^{T} C^{T},
$$

with $\Lambda_{F}(\infty)=0$. Necessary conditions for optimality state that

$$
\frac{\partial}{\partial F} E\left\{J\left(F, x\left(t_{0}\right)\right\}=E\left\{\frac{\partial}{\partial F} J\left(F, x\left(t_{0}\right)\right)\right\}=0\right.
$$

which, according to (13), implies

$$
\begin{aligned}
0 & =E\left\{\int_{t_{0}}^{\infty}-\dot{\Lambda}_{F} d t\right\}= \\
& =E\left\{\int_{t_{0}}^{\infty} 2 R F C x x^{T} C^{T}+B^{T} \lambda_{x} x^{T} C^{T} d t\right\}= \\
& =\int_{t_{0}}^{\infty}\left[2 R F C X C^{T}+B^{T} E\left\{\lambda_{x} x^{T}\right\} C^{T}\right] d t,
\end{aligned}
$$

where $X=E\left\{x x^{T}\right\}$. Performing the substitution $\lambda_{x}=2 P(t) x$, where $P(t)$ is a symmetric positive definite matrix, (11) gives

$$
2 \dot{P} x+2 P \bar{A} x=-2 \bar{Q} x-2 \bar{A}^{T} P x
$$

which is same equation represented by PLDE (8), having the periodic solution $P(t)$; moreover, the above substitution also implies that

$$
\nabla_{F} J=2 \int_{t_{0}}^{\infty}\left(R F C+B^{T} P\right) X C^{T} d t=0 .
$$

Note that the state covariance matrix $X$ satisfies the homogeneous linear matrix differential equation

$$
\dot{X}=\bar{A} X+X \bar{A}^{T} .
$$

Remark 1. An alternative way to obtain the gradient expression (16) avoids the need for the substitution $\lambda_{x}=2 P(t) x$ (which might appear somewhat arbitrary); the idea is to note that the minimization of the performance index $J=\operatorname{tr}\left(P_{0} X_{0}\right)$ is equivalent to the minimisation of

$$
J=\operatorname{tr}\left(\int_{t_{0}}^{\infty} P(t) X(t) \delta\left(t-t_{0}\right) d t\right)
$$

where $\delta\left(t-t_{0}\right)$ represents the Dirac function. Observe that the quantities $P(t)$ and $X(t)$ are different from zero only if $t \geq t_{0}$. Introducing the new Hamiltonian function

$$
H=\operatorname{tr}\left[P X \delta\left(t-t_{0}\right)-\Lambda_{P}^{T}\left(\bar{A}^{T} P+P \bar{A}+\bar{Q}\right)\right]
$$

one concludes that first order optimality conditions can be expressed again as (16).

In order to write the analytical expression of the gradient in a form suitable for numerical optimization algorithms, the integral in (16) should be computed only over a finite horizon. This can be achieved as follows. Note that (16) can be written as

$$
\nabla_{F} J=2 \int_{t_{0}}^{\infty}\left(R F C+B^{T} P\right) X C^{T} d t=
$$

$=2 \sum_{n=0}^{\infty} \int_{t_{0}}^{t_{0}+T}\left[\left(R F C+B^{T} P\right) X(t+n T) C^{T}\right] d t$

Since $x(t+n T)=\Phi_{\bar{A}}\left(t+n T, t_{0}\right) x_{0}=\Phi_{\bar{A}}\left(t, t_{0}\right) \Psi^{n} x_{0}$, we have

$$
\begin{gathered}
X(t+n T)=E\left\{x(t+n T) x(t+n T)^{T}\right\}= \\
=\Phi_{\bar{A}}\left(t, t_{0}\right) \Psi^{n} X_{0}\left(\Psi^{n}\right)^{T} \Phi_{\bar{A}}^{T}\left(t, t_{0}\right),
\end{gathered}
$$

and substituting (19) into (18) we obtain

$$
\nabla_{F} J=2 \int_{t_{0}}^{t_{0}+T}\left(R F C+B^{T} P\right) V_{\bar{A}} C^{T} d t
$$

where $V_{\bar{A}}=\Phi_{\bar{A}}\left(t, t_{0}\right) V \Phi_{\bar{A}}^{T}\left(t, t_{0}\right)$ and matrix $V$ is symmetric positive definite and satisfies the discrete Lyapunov equation

$$
V=\Psi V \Psi^{T}+X_{0} .
$$

Moreover, it is easy to show that the term $V_{\bar{A}}$ satisfies the differential Lyapunov equation

$$
\dot{V}_{\bar{A}}=\bar{A} V_{\bar{A}}+V_{\bar{A}} \bar{A}^{T}, \quad V_{\bar{A}}\left(t_{0}\right)=V .
$$

We summarise the above results in the following proposition.

Proposition 2. Let F be a constant stabilizing output feedback gain and assume that the matrices $\bar{A}(t), \quad \bar{Q}(t)$ and $X(t)$ are given respectively by $\bar{A}=A+B F C, \bar{Q}=Q+C^{T} F^{T} R F C$ and $X(t)=$ 
$\Phi_{\bar{A}}\left(t, t_{0}\right) X_{0} \Phi_{\bar{A}}\left(t, t_{0}\right)^{T}$; hence, the expressions for the performance index (2) and its gradient are given by

$$
J\left(F, X_{0}\right)=\operatorname{tr}\left(P_{0} X_{0}\right)
$$

$$
\begin{aligned}
& \nabla_{F} J\left(F, X_{0}\right)= \\
& \quad=2 \int_{t_{0}}^{t_{0}+T}\left[B^{T} P+R F C\right] V_{\bar{A}} C^{T} d t
\end{aligned}
$$

where $V_{\bar{A}}=\Phi_{\bar{A}}\left(t, t_{0}\right) V \Phi_{\bar{A}}^{T}\left(t, t_{0}\right)$ and the symmetric periodic matrices $P(t)$ and $V$ satisfy, respectively, the PLDE

$$
-\dot{P}=\bar{A}^{T} P+P \bar{A}+\bar{Q}
$$

and the discrete Lyapunov equation (DLE)

$$
V=\Psi V \Psi^{T}+X_{0}
$$

The optimization of (23) requires that the LTP system (1) is output stabilizable and, at each iteration $i, F_{i}$ belongs to the set $\mathcal{S}_{F} \subset \mathbf{R}^{m \times p}$ of stabilizing feedback gains. Formally, the optimization problem can be stated as follows:

$$
\min _{F \in \mathcal{S}_{F}} J\left(F, X_{0}\right)=\min _{F \in \mathcal{S}_{F}} \operatorname{tr}\left(P_{0} X_{0}\right)
$$

The stopping criterion, indicating the convergence to a local solution of (27) will be $\left\|\nabla_{F} J\right\|<t o l$. Finding a constant stabilizing matrix $F_{0}$ for the initialization of the algorithm is generally a non trivial problem. For this reason, the procedure indicated in (Aliev et al., 2005) for discrete LTP systems may be followed. The first step consists in considering a modified LTP system having the dynamic matrix $A_{\mu}(t)=A(t)+\mu I$; this new system will be associated to the modified optimization problem

$$
\min _{(F, \mu) \in \mathcal{S}_{F_{\mu}} \times \mathbf{R}} \operatorname{tr}\left(P_{0} X_{0}\right)+\sigma \mu^{2}
$$

where $\sigma>0$ typically takes large values. One may expect that, if $\sigma$ is sufficiently large, the iteration variable $\mu$ will converge rapidly to zero, and therefore the optimization problem (28) will coincide with the nominal problem (27) as $\mu \rightarrow 0$. Now the initialization problem consists in finding an initial value for the scalar $\mu$ such that, assuming $F_{0}=0$ for simplicity, $\rho\left(\Psi_{A_{\mu}}\right)<1$. In order to determine the gradient of $\tilde{J}$, one may observe that the problem (28) is equivalent to the minimization of the functional

$$
\begin{aligned}
J=\operatorname{tr}\left(\int_{-\infty}^{\infty} \sigma \mu^{2} \delta\left(t-t_{0}\right) d t\right)+ \\
\quad+\operatorname{tr}\left(\int_{t_{0}}^{\infty} P X \delta\left(t-t_{0}\right) d t\right),
\end{aligned}
$$

and considering the Hamiltonian function

$$
\begin{aligned}
H=\operatorname{tr}[(P X+ & \left.\sigma \mu^{2} I\right) \delta\left(t-t_{0}\right)+ \\
& \left.-\Lambda_{P}^{T}\left(\bar{A}_{\mu}^{T} P+P \bar{A}_{\mu}+\bar{Q}\right)\right]
\end{aligned}
$$

where $\bar{A}_{\mu}=A_{\mu}(t)+B(t) F C(t)$, the extremality conditions are given by

$$
\begin{gathered}
\dot{\Lambda}_{F}=-\frac{\partial H}{\partial F}=2\left(B^{T} P+R F C\right) \Lambda_{P} C^{T}, \\
\dot{\Lambda}_{P}=-\frac{\partial H}{\partial P}=\bar{A}_{\mu} \Lambda_{P}+\Lambda_{P} \bar{A}_{\mu}^{T}-X \delta\left(t-t_{0}\right), \\
\dot{\Lambda}_{\mu}=-\frac{\partial H}{\partial \mu}=-2\left[\mu \sigma \delta\left(t-t_{0}\right)+\operatorname{tr}\left(\Lambda_{P}^{T} P\right)\right],
\end{gathered}
$$

where $\Lambda_{F}(\infty)=0, \Lambda_{P}(\infty)=0$ and $\Lambda_{\mu}(\infty)=0$. Observing that $\nabla_{F} \tilde{J}$ is analogous to $(20)$, the sensitivity of the cost function $\tilde{J}$ with respect to $\mu$ can be expressed as

$$
\begin{aligned}
& \frac{\partial \tilde{J}}{\partial \mu}=2 \mu \sigma+ \int_{t_{0}}^{t_{0}+T} \operatorname{tr}\left[P \Phi_{\bar{A}_{\mu}}\left(t, t_{0}\right)\right. \\
&\left.\sum_{n=0}^{\infty} \Psi_{\mu}^{n} X_{0}\left(\Psi_{\mu}^{n}\right)^{T} \Phi_{\bar{A}_{\mu}}^{T}\left(t, t_{0}\right)\right] d t .
\end{aligned}
$$

These results are summarised in the following proposition.

Proposition 3. Let $\mathrm{F}$ be a constant stabilizing output feedback gain and assume that the matrices $\bar{A}_{\mu}(t), \bar{Q}(t)$ and $X(t)$ are given respectively by $\bar{A}_{\mu}=A_{\mu}+B F C, \quad \bar{Q}=Q+C^{T} F^{T} R F C$ and $X(t)=\Phi_{\bar{A}_{\mu}}\left(t, t_{0}\right) X_{0} \Phi_{\bar{A}_{\mu}}^{T}\left(t, t_{0}\right)$; hence, the expressions for the performance index (28) and its gradient are

$$
\begin{gathered}
\tilde{J}\left(F, \mu, X_{0}\right)=\operatorname{tr}\left(P_{0} X_{0}\right)+\sigma \mu^{2} \\
\nabla_{F} J\left(F, \mu, X_{0}\right)= \\
=2 \int_{t_{0}}^{t_{0}+T}\left[B^{T} P+R F C\right] V_{\bar{A}_{\mu}} C^{T} d t \\
\frac{\partial \tilde{J}}{\partial \mu}\left(F, \mu, X_{0}\right)=2 \mu \sigma+2 \int_{t_{0}}^{t_{0}+T} \operatorname{tr}\left[P V_{\bar{A}_{\mu}}\right] d t
\end{gathered}
$$

where $V_{\bar{A}_{\mu}}=\Phi_{\bar{A}_{\mu}}\left(t, t_{0}\right) V \Phi_{\bar{A}_{\mu}}^{T}\left(t, t_{0}\right)$ and the symmetric periodic matrices $P(t)$ and $V$ satisfy, respectively, the PLDE

$$
-\dot{P}=\bar{A}_{\mu}^{T} P+P \bar{A}_{\mu}+\bar{Q}
$$

and the DLE

$$
V=\Psi_{\mu} V \Psi_{\mu}^{T}+X_{0}
$$

\section{NUMERICAL ISSUES}

The computation of the function and its gradient involve the numerical solution of the PLDE (35), the DLE (36) and the computation of the integrals (33) and (34). We address these problems shortly in what follows.

Multiple-shooting methods to solve PLDEs have been proposed in (Varga, 2005b). Let $N \geq 1$ be an 
integer such that $\Delta:=T / N$ represents a meaningful time increment to determine the solution $P(t)$ of (35). The solution $P(t)$ at successive time moments $(k-1) \Delta$ and $k \Delta$ satisfies

$$
P_{k}=\Theta_{k}^{T} P_{k+1} \Theta_{k}+W_{k}
$$

where

$$
\begin{aligned}
P_{k} & :=P\left(t_{0}+(k-1) \Delta\right), \\
\Theta_{k} & :=\Phi_{\bar{A}_{\mu}}\left(t_{0}+k \Delta, t_{0}+(k-1) \Delta\right), \\
W_{k} & :=\widetilde{W}\left(t_{0}+k \Delta, t_{0}+(k-1) \Delta\right)
\end{aligned}
$$

with

$$
\widetilde{W}\left(t_{f}, t\right):=\int_{t}^{t_{f}} \Phi_{\bar{A}_{\mu}}^{T}(\tau, t) \bar{Q}(\tau) \Phi_{\bar{A}_{\mu}}(\tau, t) d \tau
$$

By imposing $P_{N+1}=P_{1}$, the $N$ coupled equations in (37) for $k=1, \ldots, N$ represent a discretetime backward periodic Lyapunov equation which can be solved using the algorithms proposed in (Varga, 1997). Thus, by solving the $N$ simultaneous equations (37), we determine $N$ values of the solution $P(t)$ at equidistant time instants. Since the time increment $\Delta$ can be chosen arbitrarily small, this multiple-shooting approach is certainly suited for problems with large periods and/or marginally stable dynamics when evaluating $\Theta_{k}$ and $W_{k}, k=1, \ldots, N$.

It is easy to observe that solving the DLE (36) can be cast as the solution of a discrete-time forward periodic Lyapunov equation

$$
X_{k+1}=\Theta_{k}^{T} X_{k} \Theta_{k}+G_{k}
$$

where $G_{1}=\cdots=G_{N-1}=0, G_{N}=X_{0}$ and the solution of (36) is simply $V=X_{1}$. The solution of (39) can be computed using the algorithms of (Varga, 1997). Solving the PLDE instead of the standard DLE has several advantages. First, the method used to solve both equations relies on the computation of the periodic Schur form of the matrix $\Theta_{k}$. Thus, both equations can be solved with a single reduction. Second, the computed solution $X_{k}$ represents the value of $V_{\bar{A}_{\mu}}((k-1) \Delta)$ and, as shown below, can be used efficiently to evaluate the integrals (33) and (34).

The evaluation of the gradient via the integrals (33) and (34) can be done efficiently by integrating simultaneously the PLDE (35), with $P\left(t_{0}\right)=P_{1}$, and

$$
\dot{V}_{\bar{A}_{\mu}}(t)=\bar{A}_{\mu}(t) V_{\bar{A}_{\mu}}(t)+V_{\bar{A}_{\mu}}(t) \bar{A}_{\mu}^{T}(t),
$$

with $V_{\bar{A}_{\mu}}\left(t_{0}\right)=X_{1}$ and the ordinary differential equations

$$
\begin{gathered}
\dot{G}_{F}(t)=2\left(B^{T}(t) P(t)+R(t) F C(t)\right) V_{\bar{A}_{\mu}}(t) C^{T}(t) \\
\text { with } G_{F}\left(t_{0}\right)=0 \text { and } \\
\dot{g}_{\mu}(t)=2 \operatorname{tr}\left[P(t) V_{\bar{A}_{\mu}}(t)\right], \quad g_{\mu}\left(t_{0}\right)=0 .
\end{gathered}
$$

The gradient components are

$$
\begin{aligned}
& \nabla_{F} J\left(F, \mu, X_{0}\right)=G_{F}\left(t_{0}+T\right), \\
& \frac{\partial \tilde{J}}{\partial \mu}\left(F, \mu, X_{0}\right)=2 \mu \sigma+g_{\mu}\left(t_{0}+T\right) .
\end{aligned}
$$

Table 1. Comparison of optimal closed loop performance.

\begin{tabular}{|c|c|}
\hline Control & $J$ \\
\hline open loop & 1.451 \\
LQ S.F. optimal (PRE) & 0.63 \\
LQ S.F. optimal averaged over T & 0.792 \\
LQ S.O.F. sub-optimal & 0.643 \\
\hline Difference with PRE & $2.02 \%$ \\
\hline
\end{tabular}

\section{EXAMPLE}

As a simple example, the SISO periodic system proposed in (Lovera et al., 2002), given by

$$
\begin{gathered}
A(t)=\left[\begin{array}{cc}
-1+\sin (t) & 0 \\
1-\cos (t) & -3
\end{array}\right] B(t)=\left[\begin{array}{c}
-1-\cos (t) \\
2-\sin (t)
\end{array}\right], \\
C=\left[\begin{array}{ll}
0 & 1
\end{array}\right] \quad D=0
\end{gathered}
$$

has been analyzed. For this system, constant and periodic SOF controllers have been designed, with $Q=I_{2 \times 2}, \quad R=1$. In particular, the periodic controller has the structure $F(t)=F_{0}+$ $\sum_{n=1}^{N}\left(F_{n c} \sin (t)+F_{n s} \cos (t)\right), 1 \leq N \leq 3$. Note that the system is open-loop stable (its characteristic exponents are -1 and -3 ), so we can run the optimization procedure (using the fminunc Matlab function) with the initial gain $F=0$. For the constant gain case, after 7 iterations (relative tolerance $1 \times 10^{-8}$ ) the optimal gain 0.681 was found (the stability limit for this system is $F<1.6)$. Comparing the SOF solution with the LQ full state-feedback periodic control the results reported in Table 1 were obtained. This solution may be improved considering the periodic $F(t)$ with an increasing number of harmonics. The results (see Table 2 and Figure 1) show that the first harmonic is the most relevant, as could have been expected. Besides, this numerical analysis underlines the significance of the constant feedback case, particularly in view of applications.
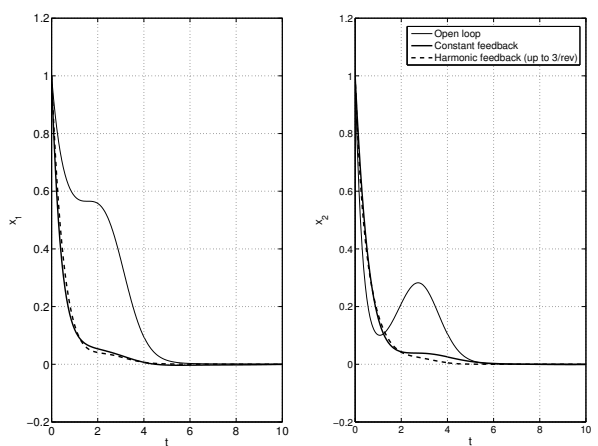

Fig. 1. State vector trajectory from $x_{0}=[1,1]^{T}$.

Finally, in order to show the effect of the choice of the covariance matrix of the initial state on the minimization of the performance index, the two approaches proposed in Section 2 have been applied: a normal distribution with zero mean and unitary standard deviation of initial conditions 
Table 2. Periodic feedback gains optimization.

\begin{tabular}{|c|c|c|}
\hline Harmonics $(N)$ & $\tilde{F}$ & Diff.with PRE (\%) \\
\hline 0 & 0.6810 & 2.1 \\
1 & {$[0.18268,0.70010,0.27482]$} & 0.05 \\
2 & {$[0.14390,0.63628,0.30402,0.06944,-0.00058]$} & 0.02 \\
3 & {$[0.13546,0.62382,0.32978,0.09989,-0.01020,-0.03783,-0.00035]$} & 0.01 \\
\hline
\end{tabular}

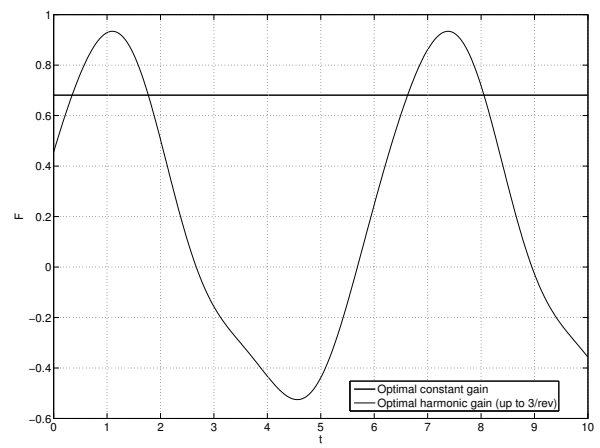

Fig. 2. Constant vs Harmonic optimal SOF Controller.

$x_{0}$ (1000 samples) has been generated; then, for each sample, the corresponding deterministic performance index has been computed using the SOF controllers optimized, respectively, for $X_{0}=I$ and $X_{0}=x_{0} x_{0}^{T}$. The results reported in Table 3 show that the assumption $X_{0}=I$ leads to a closed-loop system that behaves better in an average sense, i.e., with a smaller expected cost $\mu_{J}$ and variance $\sigma_{J}$. On the other hand, the hypothesis $X_{0}=x_{0} x_{0}^{T}$ slightly reduces the stability degree of the closedloop system but proves to be the best choice when the initial state is known exactly ( $\left.\operatorname{smaller} J_{x 0}\right)$.

Table 3. Performance evaluation with random initial conditions.

\begin{tabular}{|c|c|c|c|c|c|}
\hline$X_{0}$ & $F^{*}$ & $\mu_{J}$ & $\sigma_{J}$ & $J_{x 0}$ & s.deg. \\
\hline$I_{2}$ & 0.06813 & 1.3913 & 3.0905 & 1.33026 & 0.0067 \\
$x_{0} x_{0}^{T}$ & 0.68104 & 2.0758 & 6.5960 & 0.64271 & 0.0518 \\
\hline
\end{tabular}

\section{CONCLUDING REMARKS}

The problem of optimal static output feedback control of linear periodic systems has been considered and a novel, continuous-time approach has been proposed, which allows to deal with both stable and unstable open loop systems.

\section{REFERENCES}

Aliev, F.A., C.C. Arcasoy, V.B. Larin and N.A. Safarova (2005). Synthesis problem for periodic systems by static output feedback. Applied and Computational Mathematics 4(2), 102-113.

Ayels, D. and J.L. Willems (1993). Pole assignment for linear periodic second order systems by means of periodic memoryless output feedback. In: $32^{\text {th }}$ IEEE Conference on Decision and Control. San Antonio, Texas.
Brobrovsky, B.Z. and D. Graupe (1971). Analysis of optimal-cost sensitivity to parameter changes. IEEE Transactions on Automatic Control 16(5), 487-488.

Calise, A.J., M.E. Wasikowski and D. Schrage (1992). Optimal Output Feedback for Linear Time-Periodic Systems. Journal of Guidance, Control and Dynamics 15(2), 416-423.

Chen, M.S. and Y.Z. Chen (1999). Static Output Feedback Control for Periodically TimeVarying Systems. IEEE Transactions on Automatic Control 44(1), 218-222.

Colaneri, P., C. de Souza and V. Kucera (1998). Output stabilizability of periodic systems: necessary and sufficient conditions. In: American Control Conference. Philadelphia, USA.

Farges, C., D. Peaucelle and D. Arzelier (2006). Resilient static output feedback stabilization of linear periodic systems. In: $5^{\text {th }}$ IFAC Symposium on Robust Control Design. Toulouse.

Juan, Y.C. and P.T. Kabamba (1989). Simultaneous Pole Assignment in Linear Periodic Systems by Constrained Structure Feedback. IEEE Transactions on Automatic Control 34(2), 168-173.

Knapp, C.H. and S. Basuthakur (1972). On Optimal Output Feedback. IEEE Transactions on Automatic Control 17(6), 823-825.

Levine, W., T.L. Johnson and M. Athans (1971). Optimal limited state variable feedback controllers for linear systems. IEEE Transactions on Automatic Control 16(6), 785-792.

Lovera, M., P. Colaneri, R. Celi and S. Bittanti (2002). On the Role of Zeros in Rotorcraft Aeromechanics. In: $58^{\text {th }}$ Annual Forum of the American Helicopter Society. Montreal, Canada.

Varga, A. (1997). Periodic Lyapunov equations: some applications and new algorithms. International Journal of Control 67, 69-87.

Varga, A. (2005a). A Periodic Systems Toolbox for Matlab. In: $16^{\text {th }}$ IFAC World Congress. Prague.

Varga, A. (2005b). On solving periodic differential matrix equations with application to periodic system norms computation. In: $C D C-E C C$ '05 conference. Seville.

Varga, A. and S. Pieters (1998). Gradient-Based Approach to Solve Optimal Periodic Output Feedback Control Problems. Automatica 34(4), 477-481. 